\title{
DESIGN CALCULATION MODEL OF THREE-LAYER COMPOSITE REINFORCE CONCRETE STRUCTURES
}

The three-layer structures with a thermal insulation layer of small strength concrete keep the intermediate place between the standard reinforce concrete structures and composite constructions. The calculation model in which the cross-section of three-layer structure is substituted by I-beam homogeneous section of imaginary higher strength concrete is selected. The executed analysis have verified the possibility to use the I-beam transformed section model even for the calculation of three-layer composite reinforce concrete structural elements.

Key words: layer structure, reinforce concrete, modular ratio transformation, structural deflection, effect of shear force, bending stiffness, analysis model.

\section{Introduction}

The three-layer structure with the thermal insulation coating of low strength concrete can be ranked to the special reinforce concrete structure type. It keeps the intermediate place between the standard reinforce concrete structures and composite cross section structures with reinforce concrete external layers and the low strength and high deformable thermal insulation one in the middle. For the standard reinforce concrete structure computation, the plane-sections hypothesis can be generally used. During the deformation process according to this hypothesis, cross-sections are supposed remaining plane and perpendicular to the element axis i.e. it is supposed that the vertical shear is absent. The three-layer structures with the inner layer of low shear modulus of elasticity are calculated using the theory of composite struts. According to this theory, the action of each separate bar incorporated in composite entire strut is treated in accordance with the law of resistance of materials, especially applying the plane-sections hypothesis. The strut materials should follow the Hook's law up to the specified limit. Further than this maximum value, the plastic deformations appear.

In the case of a rigid connection all over entire length of the parts of the composite element, the final resulting structure can be considered as single common monolith element despite the fact that different parts of its cross section are produced from different materials [1]. The successive casting of the layers in the general technological cycle, producing three-layer external reinforce concrete structural part can provide monolithic nature of the section thanks to reliable connection of the external and internal concrete layers.

\section{Calculation model development}

During experimental testing of three-layer beam-type samples as well as structural parts of different concrete classes, it was observed that all three layers worked jointly up to failure. The possibility of applying the plane-sections hypothesis in calculation has been evidently proved [2]. Moreover it is supposed that no normal and inclined cracks may appear under working loads. A choice of concrete quality as well as appropriate thickness of external layers is given by this requirement. But in accordance with the presently official standard procedures for reinforce concrete structure design, values of obviously calculated deflections of the three-layered beam elements are generally underestimated [4].

Even though design calculation of the standard reinforce concrete structures before cracking occurrence could be executed using obvious structural mechanic laws commonly applied for elastic solid. However, it is necessary to consider that cross section of reinforce concrete element is not more homogeneous. In addition to concrete, the layer structure consists of steel reinforcement with elasticity modulus several times higher than the concrete one. Due to this particularity, the section should be transformed to a fictive homogeneous concrete part. The reinforcement area in this transformation is replaced by equivalent concrete area depending on the ratio of reinforcement and concrete elasticity modulus. Similarly to this practice, it would be possible to substitute the multi-layer section consisting of different concrete strength by homogeneous beam section of higher strength concrete. But the layers should be rigidly connected together and act commonly like reinforcement in normal concrete.

\footnotetext{
* J. Bujnak ${ }^{1}$, E. A. Korol², V. E. Latushkin ${ }^{2}$

${ }^{1}$ Faculty of Civil Engineering, University of Zilina, Slovakia, E-mail: jan.bujnak@fstav.utc.sk

${ }^{2}$ Moscow State University of Civil Engineering, Moscow, Russia, E-mail: korol@mgsu.ru
} 
For I-beam section, a calculated model analogous to three-layer reinforced concrete structures could be also applied. The variations of time dependent ratio of elasticity modulus of external concrete layer to internal one are taken into account by reinforce concrete beam web thickness modification.

The particularity of I-beam calculation model of a three-layered section is a slender web and wide flanges. Significant differences of elasticity modulus of outside and inside layers can exist. Due this fact corresponding design formulas as well as rules might differ from usual reinforce concrete procedures.

\section{Parametrical study based on the calculation mode}

The purpose of the following parametrical study was investigation of impacts of the high deformable inner layer, cast of low concrete strength on deflections of the overall three-layer structures. Three-layer beam specimens were $3000 \mathrm{~mm}$ long of rectangular section $160 \mathrm{~mm}$ large and $250 \mathrm{~mm}$ high. Both outside layers from structural concrete with modulus of elasticity of $15000 \mathrm{MPa}$ were $40 \mathrm{~mm}$ thick. The height of the interior layer from varying modulus of lower concrete strength was $170 \mathrm{~mm}$. Two reinforcement bars $8 \mathrm{~mm}$ in diameter were placed in the bottom exterior concrete layer. Reducing the initial value of inner layer, the concrete modular ratios under consideration in the study have varied from 1 to 150. In the case of usual one-layer rectangular section with span to height ratio $l / h>10$, shear force effect on beam deflection may be neglected, because its value is substantially inferior to that produced by bending moment [4].
A part of deflection due to shear forces, transmitted mainly by the I-beam web is given by the the formula

$$
f_{V}=\int_{0}^{1} \frac{k \cdot V(x)}{G \cdot A} d x+C_{V},
$$

where:

$C_{V} \quad$ is an integral constant, its value is zero in the case of simply supported beam;

$G$ - shear modulus of interior concrete layer;

$k$ - correction factor depending on the shape and cross section dimensions, expressed by:

$$
k=\frac{A}{I^{2}} \cdot \frac{S^{2}(z)}{b(z)} d z
$$

A - cross-sectional area,

I - second moment of area of section,

$S(z)$ - first moment of the area above the examined level, $b(z)$ - width of section part at $\mathrm{z}$ level.

Section properties of actual three-layer reinforce concrete structure can be determined more easily for the ideal I-beam. In this case, the reinforcing steel area as well as the inner layer area should be transformed to the equivalent concrete area using modular ratio. The coefficient $\mathrm{k}$ consists of expression $\frac{S^{\prime}(z)^{2}}{b_{f}}$ integrated trough the upper flange thickness, $\frac{S^{\prime \prime}(z)^{2}}{b_{f}}$ integrated trough the web height and $\frac{S^{\prime}(z)^{2}}{b_{f}}$ in the limits of the lower flange thickness:

$$
\begin{aligned}
& k=\frac{A}{I^{2}} \cdot\left\{\int_{-y_{i m}}^{-\left(y_{i m}-h_{f}\right)} \frac{S^{\prime}(z)^{2}}{b_{f}} d z+\int_{-\left(y_{i m}-h_{f}\right)}^{h-y_{i m}-h_{f}} \frac{S^{\prime \prime}(z)^{2}}{b_{f}} d z+\int_{h-y_{i m}-h_{f}}^{\left(h-y_{i m}\right)} \frac{S^{\prime \prime}(z)^{2}}{b_{f}} d z\right\}=\frac{A}{I^{2}} \cdot\left(h-y_{i m}\right)^{4} \cdot \frac{h_{f} \cdot b_{f}}{4}-\frac{\left(h-y_{i m}\right)^{2} \cdot b_{f}}{6} . \\
& \cdot\left[\left(h-y_{m}\right)^{3}-\left(h-y_{i m}-h_{f}\right)^{3}\right]+\frac{2}{b} \cdot b_{f}^{2} \cdot h_{f}^{2} \cdot \frac{b_{f}}{20} \cdot\left(h-y_{i m}-0.5 \cdot h_{f}\right)^{2} \cdot\left(h-y_{i m}-h_{f}\right)+\frac{4}{3} \cdot h_{f} \cdot b_{f} \cdot\left(h-y_{i m}-0.5 \cdot h_{f}\right) \cdot \\
& \left(h-y_{i m}-h_{f}\right)^{3}+\frac{13}{30} \cdot b \cdot\left(h-y_{i m}-h_{f}\right)^{5}+\frac{h_{f} \cdot b_{f}}{4} \cdot y_{i m}^{4}-\frac{b_{f}}{6} y_{i m}^{4} \cdot\left[y_{i m}^{3}-\left(y_{i m}-h_{f}\right)^{3}\right]+\frac{b_{f}}{20} \cdot\left[y_{i m}^{5}-\left(y_{i m}-h_{f}\right)^{5}\right]+ \\
& +\alpha \cdot A_{S} \cdot\left(y_{i m}-a\right)\left\{y_{i m}^{2} \cdot h_{f}-\frac{1}{3} \cdot\left[y_{i m}^{3}-\left(y_{i m}-h_{f}\right)^{3}\right]+\alpha \cdot A_{S} \cdot\left(y_{i m}-a\right) \cdot \frac{h_{f}}{b_{f}}\right\}
\end{aligned}
$$

However, generally the total structure deflection $f_{\text {tot }}$ consists of component $f_{M}$ produced by bending and additional part $f_{V}$ induced by shear force. Each of them depends on the shape and cross section proportions. The successive changes of the modular ratio of outside and interior concrete layers during parametrical study discovered that the rectangular form of the cross-section could be modified into I-beam shape. Thus, it is more straightforward to calculate three-layer structural part deformations using the I-beam model. Influence of shear force impact on deflection may be more appropriately investigated, because it would be possible to deal with the thinner web of equivalent I-beams.
$S^{\prime}(z)$ and $S^{\prime}(z)$ - the first moment of area of the cut section part relating to the neutral axis within the limits of the I-beam flange; $\alpha$ - the modular ratio of reinforcing steel $E_{s}$ to concrete $E_{c}$ in the transformed section;

$A_{s}$ - the reinforcing steel area in the lower flange;

$b, b_{f}$ - the width of the web/flange;

$h, h_{f}$ - the height of the web/flange.

The deflections produced by shear forces are varying with value of correction factor $k$. They are increasing, when the transformed I-beam web thickness is becoming smaller. According to our calculation model, it means that concrete modular rations are growing. 
While loading the single span beam by two identical concentrated forces, situated at the same distances from supports, the greatest shear deflection would be under load position. Its magnitude remains without changes in the region of pure bending. However, the extreme shear deflection is in the beam mid-span. Its amount can be calculated by usual structural mechanics procedures up to crack occurrences.

With growing ratios of elasticity modulus of concrete layers, the contribution of shear forces to deformations of resulting threelayer deflections are increasing significantly. For the modular ratio of outside and inner layers $E_{e x} / E_{i n}=100$, the shear force deflection would approximately equals to the value of deflection produced by bending. The common effect of shear forces is illustrated in Fig. 1. As it can be clearly seen, there is a strong growing tendency of shear forces to total deformations including bending moment contribution

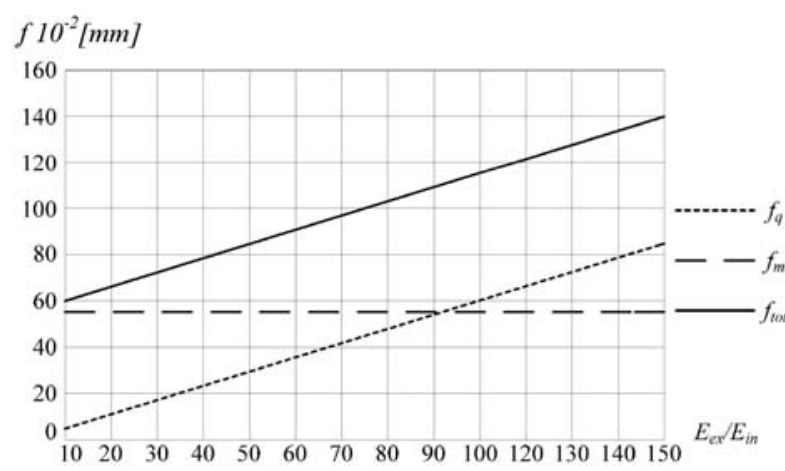

Fig. IEffect of external and internal concrete modular ratio on whole and partial deflections

In the case of normal only one-layer structure, a part of deflection due to shear forces may be negligible, because it amounts only $1.5 \%$ of total deformation. However, this amount of deflections produced by bending and shear forces might vary significantly.

In the case of modular ratio of exterior to inside layers $E_{e x} / E_{i n}=130$, the shear deflection presents $56.4 \%$ and the bending one only $43.6 \%$ (see Fig. 2). For the structures with tightly joined layers, therefore the shear forces effect on deflections should be taken into account, because from ratio $E_{e x} / E_{i n}>130$ this contribution starts to be not negligible and amounts more than $10 \%$ of the total ${ }^{\circ}$ deflection.

Design standard for concrete structures [4] expects to determine beam deflection before cracks initiation in the same way as for the solid body. For the transformed I- beam section, the following formula can be applied:

$$
f_{m}=\frac{M}{\varphi_{c 1} \cdot E_{c} \cdot I_{\text {red }}} \cdot \rho_{m} \cdot l^{2}
$$

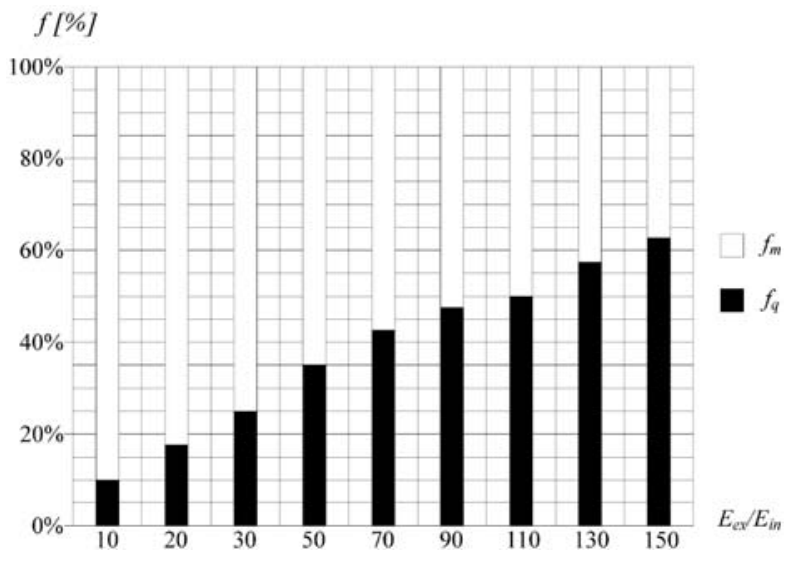

Fig. 2 Relationship between deformations produced by bending and shears effects for variable concrete modular ratio

where

$M$ - bending moment in the exanimate section;

$E_{c} \quad$ - the initial elasticity module of external concrete layer;

$I_{\text {red }}$ - second moment of area of transformed section;

$\varphi_{c 1}$ - correction factor taking into account short time concrete shrinkage effect;

$\rho_{m}$ - coefficient depending on the loading scheme (for simply supported beam loaded by two symmetrically concentrated forces applied at the distances $a$ from supports for instance

$\left.\rho_{m}=\frac{1}{8}-\frac{a^{2}}{6 l^{2}}\right)$.

l - span of the beam.

The correction factors $\varphi_{c 1}$ vary from 0.85 to 0.7 depending on the concrete class and reinforce concrete structure type. Experimental results confirm that for three-layer structural parts, the calculated bending deflection should be increased by $15 \%$ due to shrinkage rheological effects.

The stiffness of three-layer structural elements in comparison with usual ones is reduced by shear deformation action. The additional deflection due this impact can be determined from the formula

$$
f_{V}=\int_{0}^{1} \overline{V_{x}} \cdot \gamma_{x} d x
$$

where

$\bar{V}_{x} \quad$ - shear force in the exanimate point.

The factor $\gamma_{x}$ of shearing deformation produced by a short time variable loading is given by expression

$$
\gamma_{x}=\frac{1,5 \cdot V_{x}}{G \cdot b \cdot h_{0}}
$$

The resulting deflection of a reinforced concrete beam structure due to a short time live load would be: 


$$
f_{V}=\int_{0}^{1} \frac{\bar{V}}{G} \cdot \frac{1,5 \cdot V}{b \cdot h_{0}} d x
$$

Calculation procedures of beam deflection produced by shear forces usually could ignore beam flanges contribution. In our numerical model, the flange effect might be important and shear force deflection value underestimated due to transformation of real threelayer structure to an ideal I-beam. Thus, it was necessary to examine these actions by special parametrical study of real structures.

Deformations of beams with uniformly distributed load $1 \mathrm{kN} / \mathrm{m}$ were calculated alternatively by two procedures. The first method was based on a transformed I-beam section as a calculation model and the second one took into account actual three-layer design concept of three-layer structure. The beam specimen's size was the same as in previous parametrical investigation. But outside layers with the concrete modulus 12000, 24000 and $48000 \mathrm{MPa}$ were assumed. The interior concrete layer module varied from $600 \mathrm{MPa}$, which corresponds to the low thermally conductive lightweight concrete with unit mass $400 \mathrm{~kg} / \mathrm{m}^{3}$ to $30 \mathrm{MPa}$, i. e. 20 time minor.

Detail analysis of three-layer composite deformation can be found in [1]. Resulting bending deflection of three-layer reinforced concrete elements can be given as the following sum of bending deflection and shear force one:

$$
\begin{aligned}
& f=\frac{5 \cdot q \cdot l^{4}}{384 \cdot B_{t h}} \cdot\left\{1+\frac{48 \cdot(1-\lambda) \cdot k}{5 \cdot \pi^{2}}\right. \\
& \left.\cdot\left[1-\frac{2}{u^{2}}+\frac{2 \cdot \operatorname{ch} 0}{u^{2} \cdot \operatorname{ch} u}\right]\right\}
\end{aligned}
$$

where

$q \quad$ is beam uniformly distributed load;

$l$ - beam span;

$\lambda=\frac{2 \cdot B_{e x}}{B_{i n}} ;$

$B_{e x}=\frac{E \cdot h_{e x}^{3}}{12}-$ bending stiffness of the outside layer;

$B_{t h}=2 \cdot\left[E \cdot h_{e x} \cdot\left(\frac{h_{i n}}{2}+\frac{h_{e x}}{2}\right)^{2}+B_{e x}\right]-\begin{aligned} & \text { bending stiffness of } \\ & \text { three-layered section; }\end{aligned}$

$h_{e x}$ - width of outside layer;

$h_{\text {in }}$-width of interior;

$k=\frac{\pi^{2} \cdot E \cdot h_{e x}}{G_{i n} \cdot l^{2}}-\begin{aligned} & \text { multiplier depending on shear modulus of } \\ & \text { interior concrete layer } G_{i n}\end{aligned}$

$u=0.5 \cdot \pi \cdot \sqrt{\frac{1}{\lambda \cdot k}}$.
Parametrical analysis results are illustrated in Fig. 3. For actually existing modulus values of inside concrete layer, which are practically greater than $50 \mathrm{MPa}$, it can be concluded that deflections declared by calculation transforming real three-layer structure on fictive I-beam are very close to magnitudes provided by more complex procedure, considering real composition of the beam crosssections. The differences are less than $1 \%$. In addition, deflections only due to shear forces in the three layer model and I-beam model are also nearly the same, because the gaps are minor than $2 \%$. Therefore, it is possible to use the I-beam design model for calculating the three-layer structures with modular ratios $50 \leq E_{\text {ex }} / E_{\text {in }} \leq$ $\leq 600$ with sufficient accuracy (see Fig. 3).

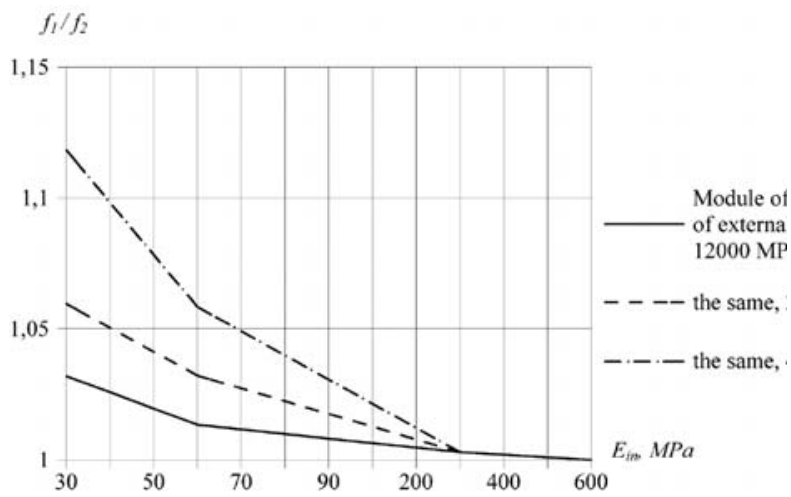

Fig. 3 Relationships between three-layer uniformly loaded elements 'deflections for different modulus of inner concrete layer

\section{Conclusion}

The executed numerical and experimental investigations have demonstrated the possibility of application of the I-beam transformed system for the calculations of three-layer composite reinforce concrete elements. It should be taken into consideration that in the case of relatively small modulus of the interior layer, the main portion of the deflections is produced by the shearing action in beam web. Therefore both shearing and bending effects should be taken into account, while calculating three-layered reinforce composite concrete structures. Resulting deflection even for layered composite reinforce concrete elements before cracking development could be determined in the same way as for the bended I-beam section. The structural theory customary rules used in these calculations should be adjusted by means of correction multipliers identified experimentally and numerically in our investigation. 


\section{References}

[1] RJANICIN, A.R.: Composite Struts and Plates, J. Stroyizdat, 1986

[2] CHINENKOV, U. V., KOROL, E. A.: To the Choice of Calculation Method for the Three-layer Lightweight Concrete External Structure, Bulletin of the construction science branch, No. 2, RAASN, 1997. pp. 423-427.

[3] CHINENKOV, U. V., KOROL, E. A.: Bended Three-layered External Constructions of Lightweight Concrete for the Second Phase of the Building Heat Insulation, Concrete and reinforced concrete, 1999, No. 2, pp. 2-5.

[4] EN 1992 Eurocode 2: Design of concrete structures, 2004. 